\title{
CHOLANGIO-HEPATITIS-A CLINICAL STUDY
}

\author{
By F. G. Hobson, D.S.O., D.M., F.R.C.P. \\ Physician to the Radcliffe Infirmary \\ and \\ J. M. RICE-OxLEY, B.M., M.R.C.P. \\ Medical Registrar, Radcliffe Infirmary, United Oxford Hospitals
}

The term cholangio-hepatitis has recently been advocated by Himsworth (1947) to distinguish a clinical disease with a well-defined morbid anatomy, but presenting a variety of clinical syndromes. The essential features of the morbid anatomy derive from infection established in the biliary tract, the severity and duration of which determine the course and gravity of the clinical disease. This infection is usually secondary to and associated with obstruction of the large bile ducts. In rare and infrequent cases infection of the tract is met with in the absence of any demonstrable obstruction. Almost invariably the organisms responsible for this infection are members of the coli-typhoid group, occasionally in association with $S$. viridans or $S$. faecalis, but in rare cases one or other of these latter organisms occurs alone. The cholangitis thus established spreads throughout the intra-hepatic portion of the biliary tract, producing an inflammatory reaction in and around the smaller bile ducts, the sequelae of which will depend upon the virulence of the organism and the duration of the infection. At one extreme there is an acute suppurative cholangitis with abscess formation, at the other a mild chronic inflammatory process which continues for years, producing a ' biliary cirrhosis,' with a varying degree of parenchymal damage, fibrosing obliterative cholangitis and secondary portal obstruction. Between these two extremes there are many intermediate cases variable both in their clinical manifestations and in the degree of parenchymal damage. In every case the cholangitis is associated with some degree of parenchymatous hepatitis, justifying the definitive description of the whole process of cholangio-hepatitis.

In considering the aetiology of this condition the two essential problems are :-

(1) What is the cause of the biliary obstruction ?

(2) How does infection reach the biliary tract?

\section{(I) Obstruction}

Excluding very rare congenital abnormalities of the tract and neoplasms, obstruction of the biliary tract arises from gallstones, biliary sludge or from surgical trauma.

(a) Gallstones. Whilst the aetiology of gallstones is still far from clear, there is little doubt that stones once formed may exist for many years without giving rise to significant symptoms, and that many such cases eventually present with symptoms due to mechanical complications or to superimposed infection. When stones and infection are confined to the gall-bladder, cholecystectomy is followed by a complete cure (Fig. I). In a proportion of cases, however, stones or debris are extruded into the main biliary passages, causing partial or complete obstruction, which may be complicated by coincident or subsequent infection of these ducts, thus providing the essential conditions for the development of cholangiohepatitis. In such cases removal of the gallbladder, even when combined with exploration and drainage of the common bile duct, may fail to effect a cure (Fig. I).

(b) Stricture. Surgical trauma is well recognized as the commonest cause of benign stricture of the common bile duct. Reviewing a series of cases of benign stricture of the common duct, Flickinger and Masson (1946) report that 186 out of 188 cases had undergone a previous operation on the biliary tract. In I Io cases symptoms of obstruction developed in the immediate postoperative period following cholecystectomy. In 22 of these cases obstructive symptoms did not develop until I to ro months after cholecystectomy following a previously uneventful convalescence. On further exploration, these cases showed an extensive obliteration of the extrahepatic ducts. Apart from surgical trauma, there is in these cases a possible alternative interpretation of the findings, namely that the disease of the gallbladder was never more than a part of the infection of the whole biliary tract, and that at the time of the initial operation, established infection of the common duct was overlooked, with the subsequent development of obliterative cholangitis. 

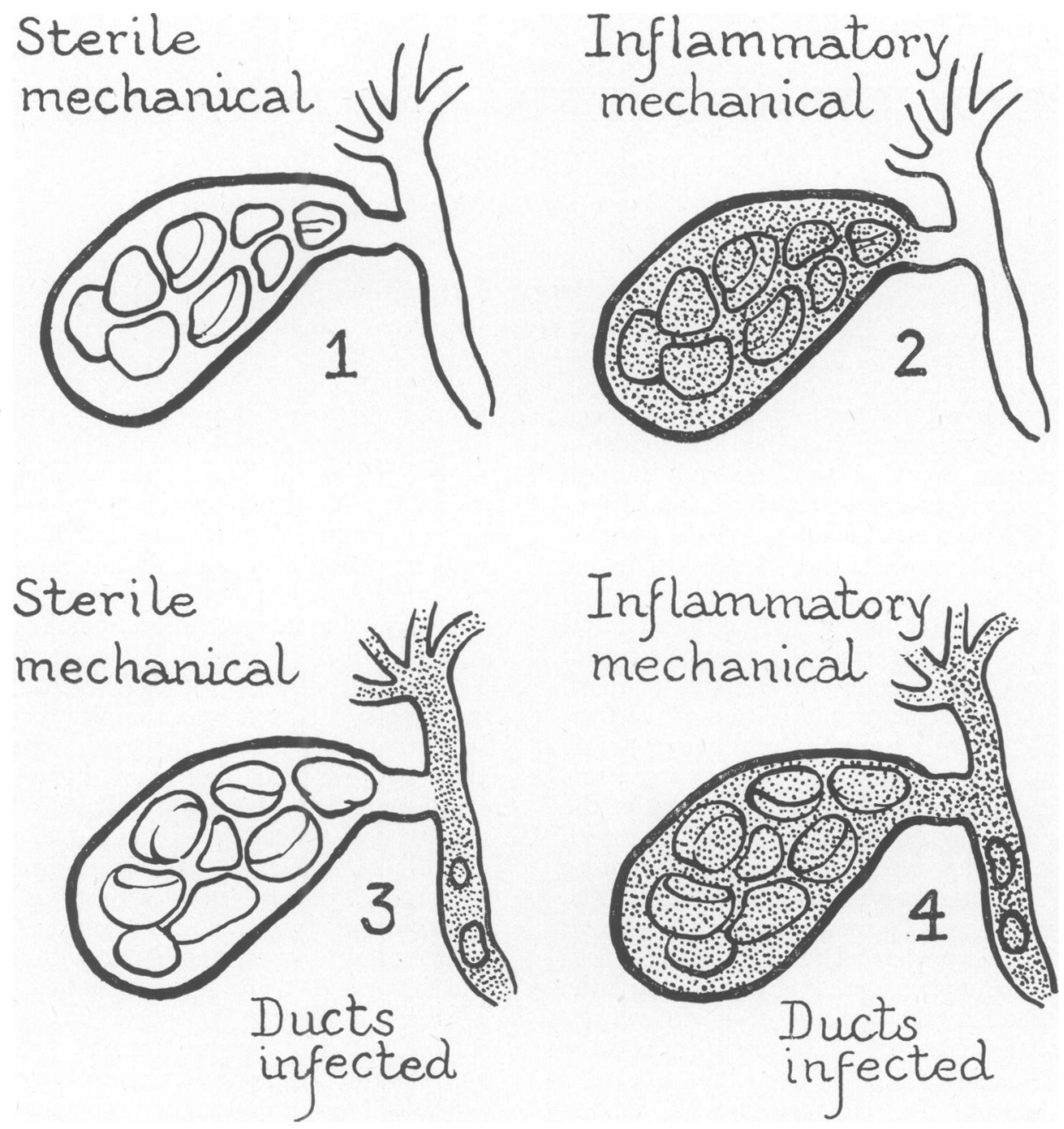

FIG. I.-I and 2. Cholecystectomy-cures. 3 and 4. Cholecystectomy even when combined with exploration and drainage of the common duct may fail to cure as the cholangio-hepatitis is untreated.

\section{(2) Infection}

How does this reach the biliary tract? The most generally accepted view is that infection reaches the gall-bladder, the biliary tract and the liver via the systemic circulation through the hepatic and cystic arteries. Wilkie's (1928) experiments indicated that such bacteria as find their way into the tract do not normally give rise to inflammation when the bile is flowing freely, but in the presence of obstruction they may give rise to cholecystitis, cholangitis and hepatitis. The organisms most frequently recovered belong to the coli-typhoid group, the members of which are frequently responsible for infections of the bacteriaemic or septicaemic type.
In past or even present-day terminology the group of cases considered in this study might have been presented under one of several clinical labels? such as Charcot's intermittent hepatic fever, cholangitis, cholangitis lenta, Hanot's biliary" cirrhosis, biliary dyskinesia (Guy, I945), or Banti's syndrome, always however accepting that this syndrome is more often the product of degenera-0 tive hepatic fibrosis of non-inflammatory origine It is suggested that the term cholangio-hepatitis is more accurate and better justified by the demon? strable features of the morbid anatomy.

\section{Clinical Cases}

The clinical material consists of $3 \mathrm{I}$ cases ad $\stackrel{\mathbb{D}}{\mathbb{D}}$ 
mitted to the general medical or surgical wards of the Radcliffe Infirmary over a period of about 10 years. Every case has been a case of cholelithiasis. Three of these cases died shortly after admission without operation. In the remaining 28 cases the gall-bladder was removed for cholelithiasis, with cholecystitis. In each of these 28 cases there was, therefore, clear evidence of disease of the biliary tract, and an opportunity was presented in each case for inspecting the main bile ducts, and for obtaining from them evidence of the presence of infection by direct culture.

In 27 of these 28 cases cholecystectomy completely failed to relieve the symptoms from which the patient was suffering, as the cholelithiasis and cholecystitis were no more than part of a disease of the whole biliary tract, and the post-operative symptoms were identical with those present before operation. These patients were, in fact, cases of cholangio-hepatitis, whose symptoms returned within a few weeks or a few months of their discharge from hospital.

Symptoms and signs. There are four characteristic symptoms of cholangio-hepatitis.

(I) Attacks of upper abdominal pain.

(2) Vomiting.

(3) Jaundice.

(4) Rigors, chills and shivering attacks.

All these symptoms and signs were present in each of the 27 cases mentioned above prior to cholecystectomy, and in each case the same symptoms and signs recurred post-operatively in varying frequency and in different combinations, no single case having failed to present jaundice in some or all of the relapses. The post-operative clinical diagnosis has been confirmed by the recovery of infected bile, yielding $B$. coli on culture in 24 cases. In 4 cases only does the diagnosis rest upon clinical evidence unconfirmed by culture of the bile.

The clinical picture here presented is not, however, quite so simple as this bald statement of the general facts might suggest. Himsworth has suggested the clinical subdivision of cholangiohepatitis into three syndromes, namely:-the acute suppurative, which proceeds to a rapid termination; subacute cholangio-hepatitis, comprising many cases of moderate severity with recurrent symptoms compatible with survival for many months or even two or three years; and chronic cholangio-hepatitis, comprising cases which may endure for years with symptoms of vague ill-health. Himsworth is, however, at pains to emphasize that these broad distinctions, while convenient, do not imply the existence of separate and distinct entities but simply degrees of one process, the morbid anatomical sequelae of which are progressive but variable in their severity, a process which will produce clinical pictures of some complexity and variety. Our cases provide ample evidence in support of Himsworth's main thesis, and we have been able to trace a common historical pattern in the development of the process. This can be summarized as followed:-

Phase I. Gastrointestinal disturbances, essentially mechanical in origin and attributable to the presence of gall stones. The duration of this phase has varied from a few weeks to more than 20 years.

Phase 2. Gastrointestinal disturbances, with symptoms and signs attributable to infection of the biliary tract, a cholangio-hepatitis. These symptoms and signs invariably comprise pain, vomiting, jaundice and rigors. Depending upon the severity of the symptoms, this phase may cover a period of a few days to a few months, even to a year, before a surgical opinion is sought.

Phase 3. Cholecystectomy.

Phase 4. The return of precisely those symptoms and signs for which the cholecystectomy was performed. This phase is marked by relapses at intervals of a few weeks or a few months, with symptoms and signs of variable severity, comprising pain, vomiting, jaundice and rigors. Jaundice is a variable feature of the relapses. Evidence of parenchymal hepatitis is dependent upon the severity and duration of the infection.

Phase 5. In which a progressive biliary cirrhosis leads on to liver failure, portal obstruction, ascites and oesophageal varices.

The 3 I cases fall naturally into four main syndromes, which differ somewhat from Himsworth's classification.

\section{The Four Clinical Syndromes}

\section{(I) Pyrexia of Unknown Origin (2 cases)}

I. This case (female, age 76 ) was remarkable in that minute and detailed investigations over a period of two years failed completely to reveal any cause for recurrent attacks of high, remittent pyrexia of variable duration. During these attacks, which lasted up to several weeks, there was a curious absence of any constitutional disturbance, the patient insisting upon her competence to conduct her normal activities in spite of an evening pyrexia of $103^{\circ} \mathrm{F}$. A transient jaundice, apparent as a terminal event, provided the first and solitary indication of any lesion of the liver or biliary tract. At autopsy there were multiple liver abscesses yielding pure cultures of $B$. coli, with diffuse 'biliary cirrhosis'; the in- 
testines contained multiple calculi which had obviously been only recently discharged from the dilated and chronically inflamed common duct.

2. Male (age 79) giving a history of one year's pain across the chest, unrelated to exertion or gastrointestinal function. Six weeks prior to admission he began to experience intermittent rigors, with progressive loss of appetite. He started on a holiday but his rigors recurred more frequently. There was no jaundice, the urine was sterile and contained no albumen, sugar or bile. Abdomen-

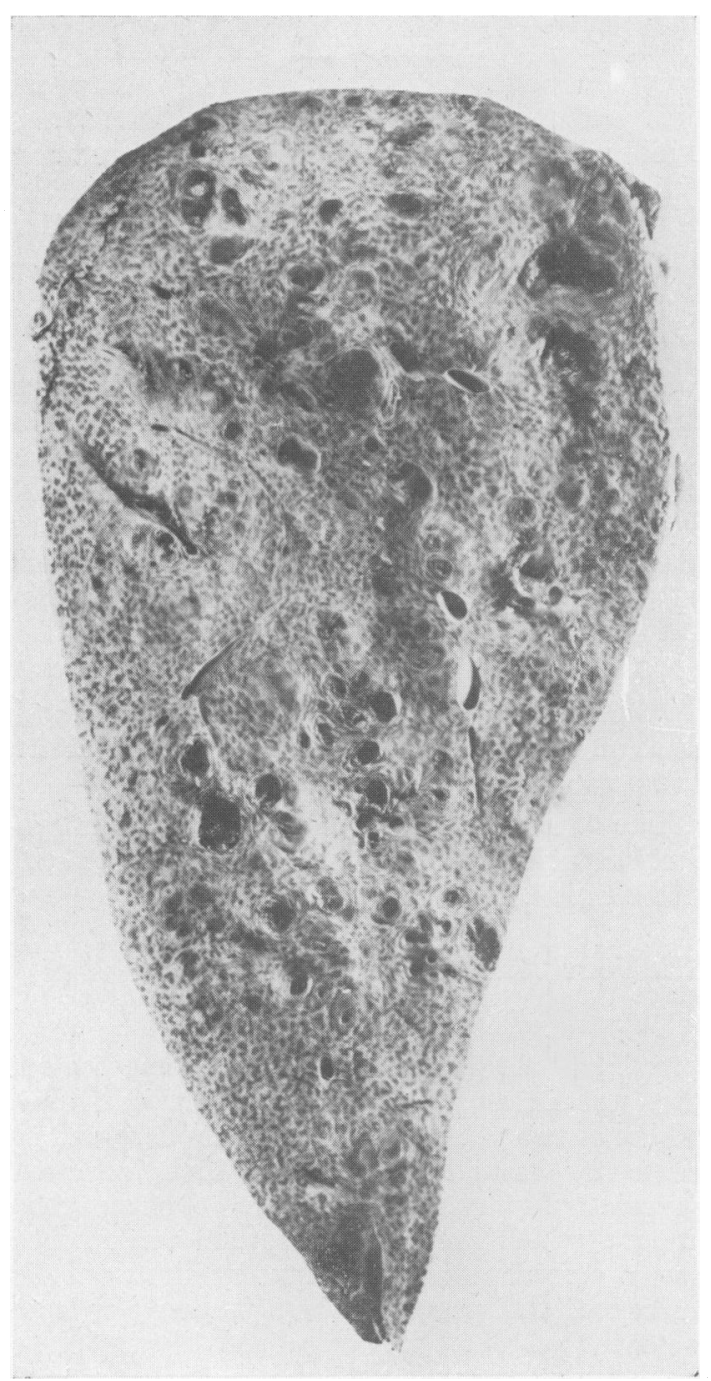

FIG. 2.-Case E.G. Macroscopic view of liver, which weighed $1,700 \mathrm{gm}$. The section is taken from: the right lobe and shows multiple ahssess cavities at different stages of development, but all arising in the portal tracts as a result of suppurative cholangitis.

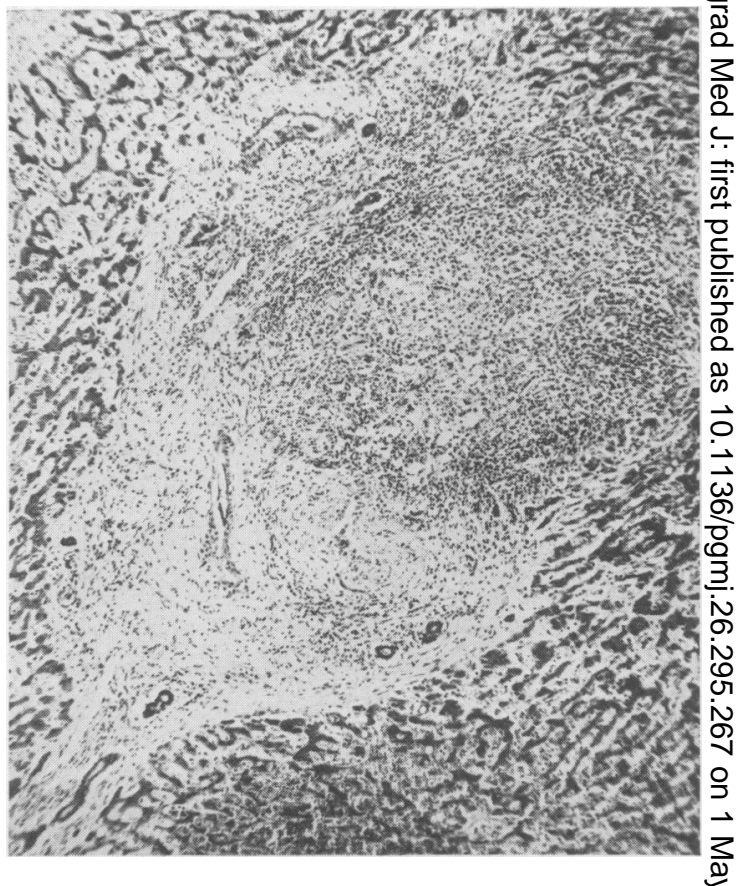

FIG. 3.-Case E.G. Microscopic view of one portaltract. A small abscess is shown in an early stage of organization. The bile duct from whichoito arose has been obliterated; a tributary duct (tep left) is only mildly affected, and there is evidefeo of duct regeneration in the neighbourhood of the abscess. The tract shows fibrosis, much of which is recent; the artery (left centre) shows degenerative changes. The shrinkage of liver cells is largely thex result of post-mortem degeneration.

no localizing signs. Blood culture-B. coli in pure culture. Blood bilirubin-I.I mgm. per cent

Operation. Cholecystectomy. Three calculi and much debris in gall-bladder. Aspiration of the common duct yielded $B$. coli in pure culture:The duct was explored but no obstruction found the duct was drained. After a full course of sul-8 phonamides, convalescence was uneventful and recovery apparently complete.

These two cases illustrate the fact that jaundices may be notable by its absence even when there is an acute virulent infection in the biliary tract. In the first case jaundice was apparent only as a transient terminal episode. The second case was remarkable as being the solitary example in thew series in whirh a positive blood culture waso obtained.

\section{(2) Suppurative Cholangitis (4 cases)}

I. Female (aged 74) admitted moribund, heavily jaundiced. At autopsy there were multiple livero abscesses and 'biliary cirrhosis'; $B$. coli was? grown in pure culture from the abscess cavities $\stackrel{\square}{Q}$ 
There was a history of symptoms suggestive of cholecystitis of about I2 months' duration, followed by an acute, sudden exacerbation three days before death.

2. Male (age 80). No clear history of previous ill-health other than dysuria of recent onset. Admitted jaundiced and moribund. Liver abscesses yielding $B$. coli in pure culture and biliary cirrhosis (Figs. 2 and 3 ).

3. Female (age 39) admitted with a history of two attacks of pain, vomiting, jaundice and rigors, since cholecystectomy for cholelithiasis three months prior to admission. During cholecystectomy the common bile duct had been inadvertently divided and repaired by suture over an indwelling T-tube. Duodenal intubation yielded a pure culture of $B$. coli.

A laparotomy was performed on this patient but no evidence of any gross obstruction was discovered either in the common or hepatic ducts. Repeated attempts to sterilize the biliary tract with sulphonamides were unsuccessful, as tested by duodenal aspiration. Death occurred in an acute septicaemic exacerbation shortly after discharge from hospital This was the solitary case in the series in which recognized surgical trauma occurred.

4. Male (age 66). This patient gave a sevenyear history of attacks of biliary colic, with a severe exacerbation of symptoms two months prior to admission. Cholecystectomy was performed but the patient died two weeks after operation. At autopsy a ' missed' calculus was discovered in the common duct, together with a large abscess in the liver, yielding pure $B$. coli on culture.

\section{(3) Subacute (or Chronic) Cholangio-Hepatitis (22 cases)}

In this major group it is difficult to differentiate clearly between subacute and chronic cholangiohepatitis. No patient in this group is prepared to regard his sufferings as mild, and relapses have occurred over a number of years. In every case the gall-bladder had been removed for cholelithiasis with cholecystitis. The first relapses occurred usually within three months of operation, in some cases even within a few days or weeks of operation, and major relapses have followed at intervals varying from six weeks to nine months. In one case three or more rigors a week continued for many months after operation.

Of these 22 cases three have had two further operations when calculi or biliary sludge were discovered in the common duct, and $B$. coli found on culture. Four cases have had one further operation on the common duct, in each case calculi being found in the duct. One of these cases had relapsed after a successful post-operative sterilization of the tract with sulphonamides, confirmed by the recovery of a sterile bile by duodenal intubation. Nine cases are leading fairly healthy lives, interrupted at unpredictable intervals by relapses of shorter or longer duration and varying severity. Five cases responded successfully to sulphonamide therapy, success being judged by the fact that a sterile bile was recovered on the completion of treatment, the bile having yielded $B$. coli on culture before treatment. These cases have remained symptom free, as confirmed by a follow-up enquiry, the shortest five years, the longest ten years after treatment. It is reasonable to suppose that in these five cases there was no significant postoperative obstruction of the biliary tract and that the cholangio-hepatitis was cured by eradication of the infection. One case has responded successfully to combined sulphonamide and streptomycin therapy, but sufficient time has not yet elapsed to regard the patient as cured.

Two illustrative cases are given in detail.

I. Female (age 70). I916 (Phase I). Aged 50, she experienced an attack of upper abdominal pain diagnosed as gallstones. She was dieted and remained relatively symptom free until 1927 , when attacks of pain recurred at variable intervals.

1936 (Phase 2). Recurrent attacks at short intervals of abdominal pain, vomiting, jaundice and chills lasting five weeks. Gall-bladder palpable.

I936 (Phase 3). Cholecystectomy-gallstones.

(Phase 4.) Four months after operation she experienced an acute attack of upper abdominal pain with fever and shivering. These attacks recurred at intervals of a few months and were frequently associated with transient jaundice.

I939. Admitted with slight jaundice, recovering from a recent relapse. Duodenal intubation showed that "all specimens of bile contain large numbers of pus cells, coliform bacteria and streptococci. Culture-profuse growth of B. coli.' She was treated with a full seven-day course of sulphapyridine, following which duodenal intubation revealed sterile bile. The patient, now $8 \mathrm{I}$, has been symptom free for ten years.

2. Male (age 63). 1936 (Phase I). Began to have attacks of epigastric pain about an hour after meals, relieved by alkalis and occasionally by vomiting. These attacks continued at variable intervals.

I 946 (Phase 2). He experienced a severe attack of epigastric pain radiating to the scapulae with vomiting, shivering and transient jaundice.

1947. 'Two similar attacks with rigors. Later admitted to hospital with jaundice and a tem- 
perature of $\mathrm{IOI}^{\circ}$; both jaundice and fever subsided.

(Phase 3.) Cholecystectomy. The common duct was found to be dilated; it contained one large stone with much debris, and was drained. Culture of the bile showed B. coli and scanty Ps. pyocyanea. Penicillin, but no sulphonamide, was given postoperatively. A post-operative cholangiogram showed no obstruction of the duct.

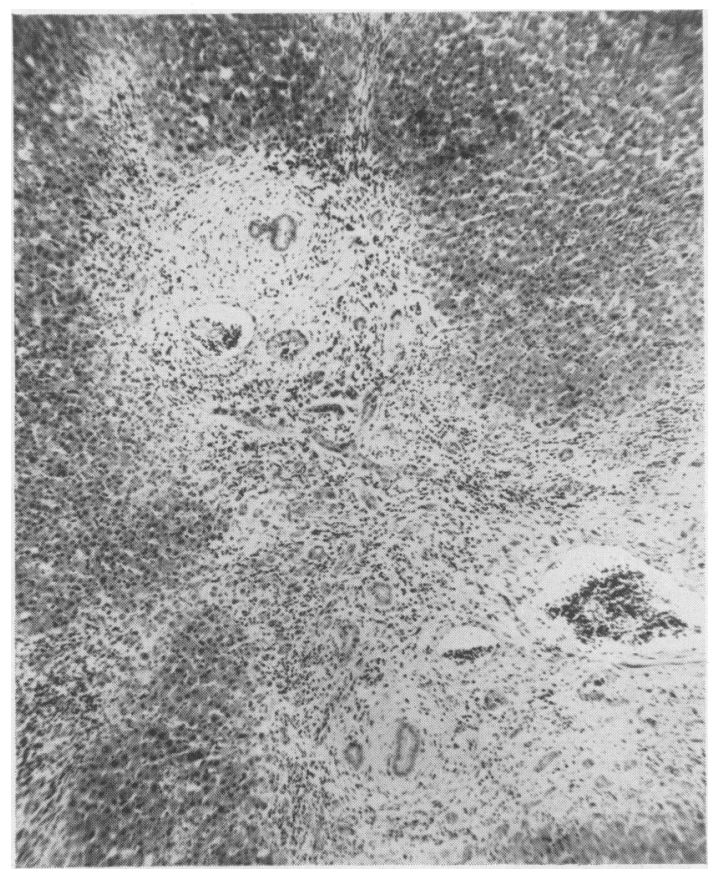

Fig. 4--Case R. J. Microscopic view of liver (biopsy specimen). The portal tracts show fibrosis and small round cell infiltration; much of the fibrous tissue lies in concentric rings about the bile ducts.

1948, January (Phase 4). Severe recurrence of pain with vomiting and jaundice. Admitted and given full course of sulphamezathine and penicillin.

1948, November. Further mild and severe attacks of pain occurred together with vomiting and jaundice. Investigations showed:-

Duodenal intubation-pure culture of B. coli.

Blood chemistry-serum bilirubin $2.0 \mathrm{mgm}$.

Serum phosphatase 27 units.

Serum protein, total 6.4 gr.; albumin 3.4 gr., globulin $3.0 \mathrm{gr}$.

Serum flocculation tests negative.

1948, December. Choledocho-duodenostomy. Common duct explored; found dilated with constriction at ampulla and containing five large cholesterol stones with much debris. $B$. coli on culture. (Liver biopsy Fig. 4.) Post-operatively a full course of sulphamezathine was given. free.

I949, July. The patient was well and symptom

\section{(4) Banti's Syndrome (3 cases)}

I. Female (age $5^{8}$ ) admitted with history of more than 20 years of gastrointestinal disturbances, 'bilious' attacks and vomiting suggestive of cholelithiasis. Following admission with severe cholaemia and biochemical evidence of severe parenchymal damage, she recovered and a cholecystectomy was performed in March I948, a small, withered gall-bladder being removed together with a large stone found in the common duct, the bile yielding $B$. coli in pure culture. In October 1948 she had a severe haematemesis; examination showed an enlarged spleen and palpable liver, while oesophageal varices were easily demonstrated with a barium swallow.

Splenectomy was performed.

2. Male (age 37). 1932. Onset of jaundice and fever with an enlarged liver; a cholecystogram showed a non-filling gall-bladder and he continued to experience mild recurrent attacks until 1934, when the attacks became more severe ando were associated with rigors. These attacks oc $=0$ curred at variable intervals until 1942, when he was admitted for cholecystectomy. The gallbladder showed white mucoid contents, the common duct was unusually small, possibly a congenital abnormality, and contained a number of pigment stones. Macroscopically the liver appeared to be normal. There was no evidence of haemolytic jaundice. In 1943 he was readmitted for recurring attacks of pain, vomiting, fever and jaundice. Duodenal intubation yielded a culture of $B$. coli and $S$. viridans. Biochemistry: proteins, total, 6.6 gr., albumen 3.8 gr., globulin 2.5 gr., plasma phosphatase 32 units. He was treated with hexamine, but in 1945 presented again with persistent mild jaundice with episodic deepening. The liver was easily palpated and the lower pole of spleen was just palpable. Duodenal intubation yielded a culture of $B$. coli. Plasma proteins, total, 7.9 gr., albumen 4.4 gr., globulin 3.0 gr., plasma phosphatase 54 units. He was treated with successive courses of sulphathiazole, sulphadiazine and sulphanilamide without benefit; following each course duodenal intubation yielded $B$. coli in pure culture. In 1946 a laparatomy was performed. Macroscopically the liver showed a coarse nodular fibrosis, no stones were felt in the common duct and choledochoduodenostomy was deemed impracticable. In 1948 ascites first appeared and repeâted aspiration was necessary until his death later in the year (see Fig. 5). 


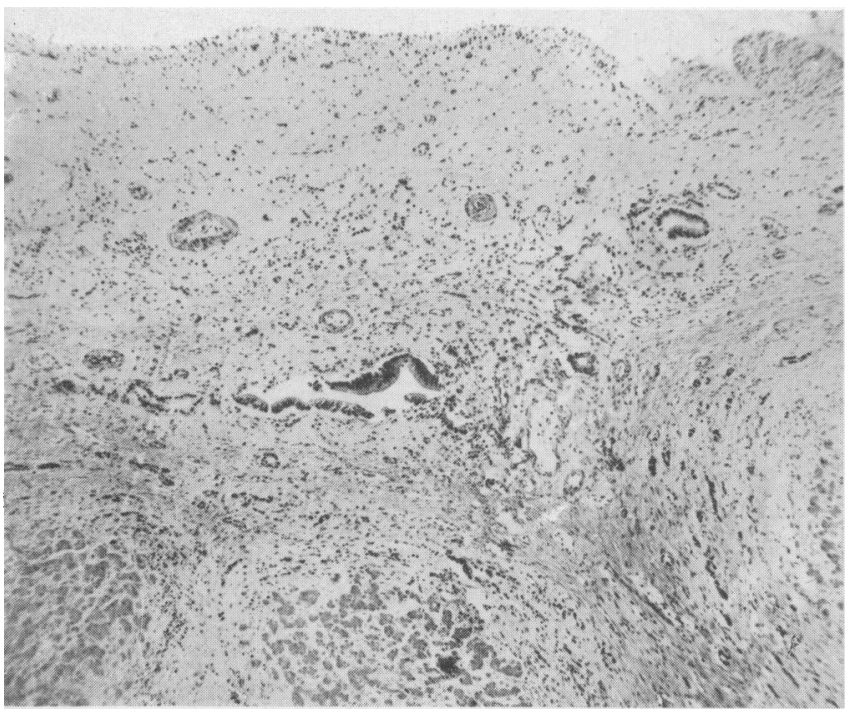

FIG. 5.--Case W.P. Microscopic view of liver. The section is taken from the wall of a large bile duct, the lumen of which crosses the upper part of the picture. The mucosa has been destroyed and the duct is lined by granulation tissue with much fibrosis in the deeper layers; here also regenerated bile ducts are seen. Three islets of liver tissue, surrounded by thick fibrous tissue, are seen at the bottom of the picture, and indicate the advanced degree of biliary cirrhosis present in this case.

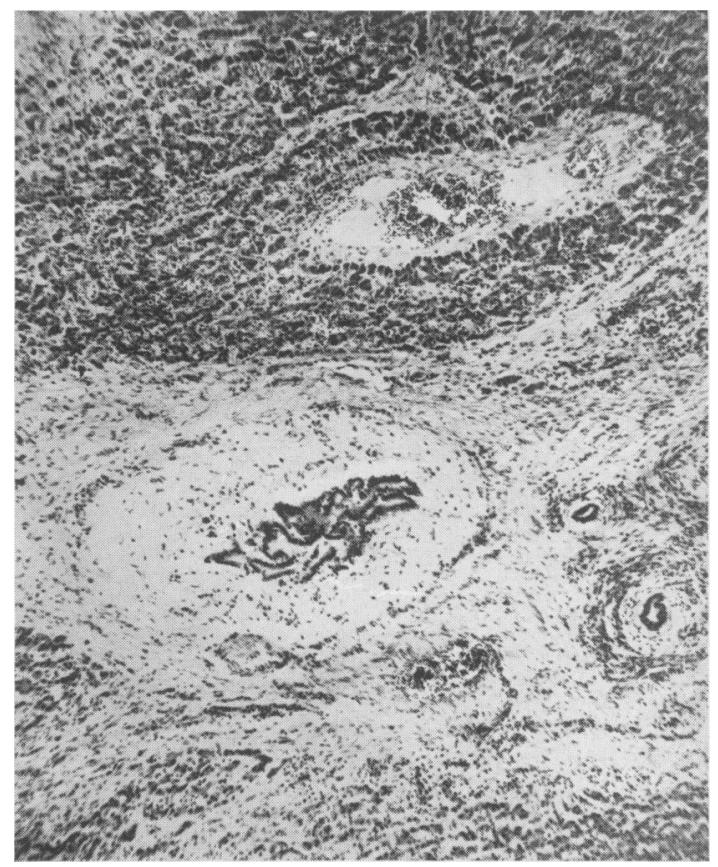

FIG. 7.-Case G.E. Microscopic view of liver, showing a portal tract close to the wall of one of the abscess cavities. This demonstrates the very marked fibrosis in the tract, especially about the ducts, as a result of long-standing inflammation. The distortion of the mucosa in the large duct is an artefact probably due to post-mortem desquamation.

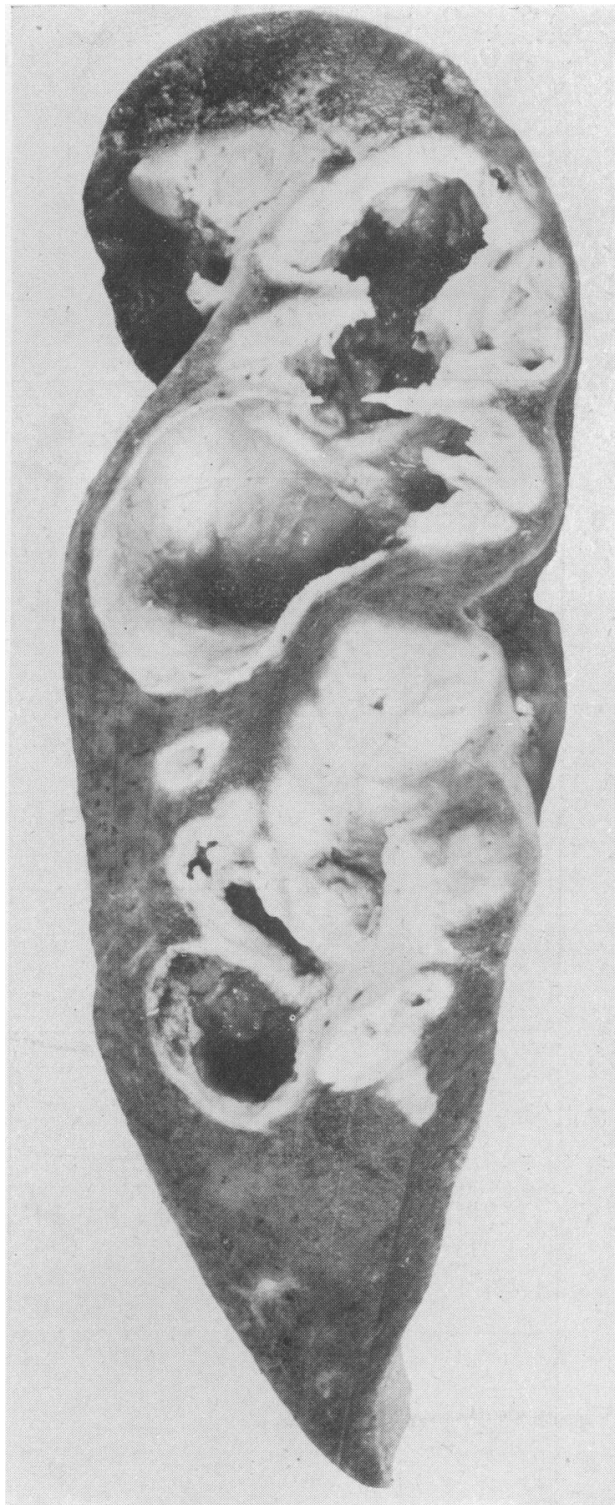

FIG. 6.-Case G.E. Macroscopic view of liver, which weighed 2,135 gm. In this section from the left lobe, large abscess cavities are the most striking feature.

3. Male (age 35). Cholecyst-gastrostomy was performed for recurrent attacks of jaundice and fever at the age of 29 , but following a temporary remission of his symptoms readmissions for recurrent attacks of jaundice and fever were necessary over a period of five years until his death with parenchymal failure, splenomegaly and ascites. Over the period of readmissions there was biochemical evidence of progressive parenchymal damage. At autopsy the liver was 


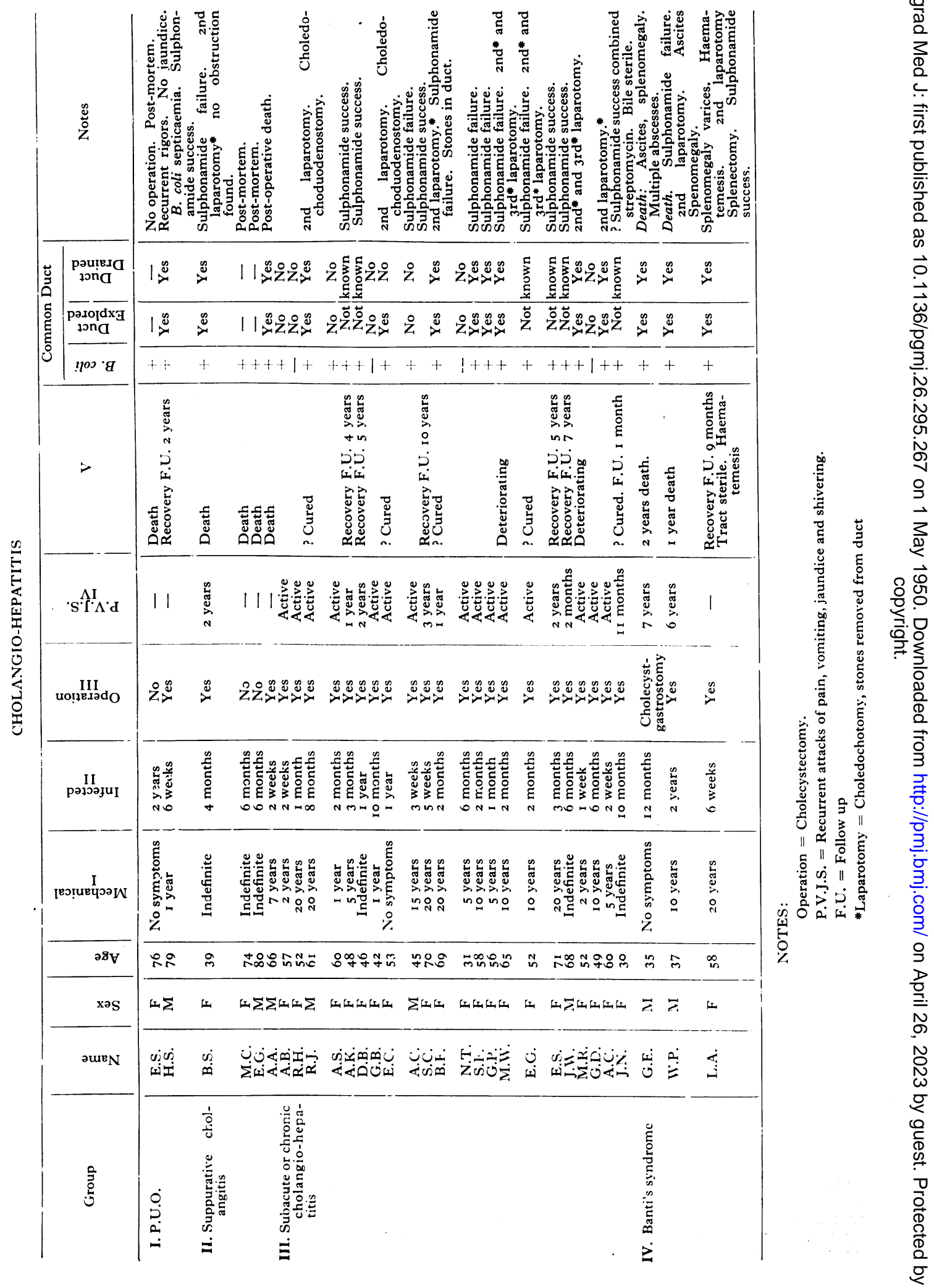


grossly enlarged and showed a chronic cholangiohepatitis with biliary cirrhosis and multiple abscess cavities yielding $B$. coli in pure culture. 'The case is one of unusual interest in that upon a protracted chronic cholangio-hepatitis there supervened the acute suppurative phase with abscess formation (see Figs. 6 and 7 ).

Summarizing briefly the main features of cholangio-hepatitis as illustrated by the cases reported, it is apparent that it is a major disease of the biliary tract, the presence of which may escape detection because the diagnostic and therapeutic efforts are concentrated upon and diverted to the minor, more obvious, coincident and provocative disease of the gall-bladder which is no more than a part of the pathological process. The course of the disease may be fulminant or intermittent. If unrecognized and inadequately treated it will inevitably pursue its relentless course with minor or major exacerbations, giving rise to a progressive biliary cirrhosis, hepatitis and parenchymal failure, terminating in a Banti's syndrome with ascites or haemorrhage.

\section{Discussion}

Considering the uniformity of the clinical picture presented by this condition, especially in its fourth post-operative stage, it is remarkable that more attention has not been paid to it in the past. This neglect is perhaps due to its comparative rarity, but an investigation carried out upon all patients undergoing cholecystectomy for cholelithiasis at the Radcliffe Infirmary during the years 1943 to 1947 showed that out of 163 cases 8 showed indubitable evidence of cholangio-hepatitis. A condition of such severity occurring in 5 per cent. of patients undergoing cholecystectomy obviously demands serious consideration.

Diagnosis of the existence of cholangio-hepatitis prior to cholecystectomy is admittedly difficult. The cardinal symptoms of pain, vomiting, jaundice and shivering are suggestive but not diagnostic, as they may occur in cases in which the inflammation is confined to the gall-bladder, with a transient hepatitis. Post-operatively the recurrence of these symptoms, singly or in combinations, is very suggestive of the existence of cholangio-hepatitis; shivering attacks and jaundice are of particular significance and almost diagnostic. In general biochemical and haematological investigations are unhelpful in establishing the diagnosis, and the only indubitable evidence is the isolation of $B$. coli or other organisms from the bile, either at the time of operation or by duodenal aspiration before or after operation.

Analysis of the 27 patients with cholangio- hepatitis reported in this paper show that in 9 cases the common bile duct was neither explored nor drained, and that post-operative sulphonamide therapy was only employed in six. In only I case was there any major surgical trauma to the common duct. In the light of this analysis and bearing in mind that obstruction and infection are the two factors of paramount importance in the aetiology of cholangio-hepatitis, we would suggest that the following measures should be carried out in order to diagnose and prevent this very serious complication of cholelithiasis.

I. At the time of cholecystectomy, especially in the presence of obstruction or any other pathological abnormality in the common duct, the unique opportunity of obtaining cultures from bile in the duct should not be missed.

2. Bearing in mind that experienced surgeons are of the opinion that neither by inspection nor palpation is it possible to express a confident opinion that no obstruction to the duct exists, exploration of the common duct and dilatation of the sphincter of Oddi should be carried out in a high proportion of cases submitted to cholecystectomy.

3. In cases of proven obstruction or infection of the common duct the drainage tube should not be removed until (a) there is definite evidence of patency of the duct; (b) culture has shown the bile to be sterile.

4. In cases of obstruction and infection of the common duct operation should be combined with and followed by a course of chemotherapy, sulphonamides and streptomycin being the obvious drugs of choice.

In our opinion the introduction of these measures would greatly improve the results of treatment in these difficult cases, though they would not, of course, prevent the development of cholangio-hepatitis following gross operative trauma to the bile ducts. This disaster continues to present one of the most difficult problems of biliary surgery, and no wholely satisfactory treatment is as yet available; as Flickinger and Masson (I946) remark: ' Traumatic stricture of a bile duct is a tragic accident, which entails untold misery for the patient, presents a formidable task to the surgeon and even with the best treatment attempts at reconstruction are followed by a high mortality.'

\section{Summary}

The pathological and clinical manifestations of cholangio-hepatitis are described, and it is emphasized that cholecystectomy is not a cure for the condition. 
A series of $3 \mathrm{I}$ cases of cholangio-hepatitis is presented. Four major clinical syndromes are illustrated in this series of cases :

I. Pyrexia of unknown origin

2. Suppurative cholangitis

3. Subacute (chronic) cholangio-hepatitis

4. Banti's syndrome

with a common historical pattern which is described. Duodenal intubation is an invaluable aid in diagnosis, and moreover can provide evidence of the success or failure of surgical or bacteriostatic therapy employed to suppress the infection in the biliary tract.

It is suggested that adequate drainage of the common duct, in association with vigorous chemo- therapy, are the essential features in the management of the condition.

We wish to record our gratitude to Dr. C. Bartley for assistance with the records, to Dr. A. H. T. Robb-Smith for help and criticism, to Dr. R. I. K. Elliott for the preparation of the pathological specimens and illustrations, to Dr. R. H. Vollum for the bacteriological investigations, to Mr. G. Higgins for the biochemical data and to our surgical colleagues on the staff of the United Oxford Hospitals, Radcliffe Infirmary, for permission to use their records and to study the cases under their charge.

We also wish to express our thanks to Professor L. Witts for a grant from the Nuffield Research Fund to meet secretarial and postal expenses.

\section{BIBLIOGRAPHY}

HIMSWORTH, H. P. (1947), 'The Liver and its Disease.'

FLICKINGER, F., and MASSON, J. C. (1946), Surg. Gynec. Obstet., 83, 24.

WILKIE, A. S. (1928), Brit. F. Surg., 15, 450.

WILKIE, A. S. (1929), Ibid., 16, 214 .

GUY, C. C. (1945), Indust. Med., 14, 181. 